\title{
Distribution of NMR Relaxations in a Random Heisenberg Chain
}

\author{
T. Shiroka, ${ }^{1,2}$ F. Casola, ${ }^{1,2, *}$ V. Glazkov, ${ }^{1,3}$ A. Zheludev, ${ }^{1}$ K. Prša, ${ }^{1,2}$ H.-R. Ott, ${ }^{1}$ and J. Mesot ${ }^{1,2}$ \\ ${ }^{1}$ Laboratorium für Festkörperphysik, ETH Hönggerberg, CH-8093 Zürich, Switzerland \\ ${ }^{2}$ Paul Scherrer Institut, CH-5232 Villigen PSI, Switzerland \\ ${ }^{3}$ P.-L. Kapitza Institute for Physical Problems RAS, 119334 Moscow, Russia \\ (Received 3 December 2010; revised manuscript received 7 March 2011; published 1 April 2011)
}

\begin{abstract}
NMR measurements of the ${ }^{29} \mathrm{Si}$ spin-lattice relaxation time $T_{1}$ were used to probe the spin- $1 / 2$ random Heisenberg chain compound $\mathrm{BaCu}_{2}\left(\mathrm{Si}_{1-x} \mathrm{Ge}_{x}\right)_{2} \mathrm{O}_{7}$. Remarkable differences between the pure $(x=0)$ and the fully random $(x=0.5)$ cases are observed, indicating that randomness generates a distribution of local magnetic relaxations. This distribution, which is reflected in a stretched exponential NMR relaxation, exhibits a progressive broadening with decreasing temperature, caused by a growing inequivalence of magnetic sites. Compelling independent evidence for the influence of randomness is also obtained from magnetization data and Monte Carlo calculations. These results suggest the formation of random-singlet states in this class of materials, as previously predicted by theory.
\end{abstract}

Random variations of the exchange coupling constant $J$ in ferro- and antiferromagnetic systems (bond randomness) can have a profound effect on their magnetic properties. An innovative scheme for studying random spin-1/2 Heisenberg chains (RHCs) was developed already in 1979 by Dasgupta and Ma [1]. Their real-space renormalization group method was extended by Fisher [2] and later employed for a much larger variety of problems related to quenched disorder in quantum magnets. Among these are chains with random ferro- and antiferromagnetic couplings [3], disordered spin- 1 chains and spin-1/2 ladders [4,5], and Heisenberg magnets in two and three dimensions [6], as well as dilute spins in doped semiconductors [7]. At the core of the real-space renormalization group method is the so-called decimation procedure. For RHCs it involves an iterative suppression of the degrees of freedom of the most strongly coupled spins via the formation of "frozen" singlets. Fisher [2] has shown that this procedure converges to a universal fixed point. The resulting ground state, characterized by spins coupled at all possible distances and energy scales, is also known as the random-singlet (RS) state. At low temperatures, only excitations of the most weakly bound singlets are expected to be relevant [8]. As a result, regardless of the microscopic details of the disorder, a large class of bond-disordered systems share universal low-temperature and low-energy properties. In recent years, besides providing predictions for bulk properties, new theoretical work has started to address also the problem of dynamical observables [8,9].

To date only a few good realizations of RHCs have been achieved. Some early examples [10] were later found to have additional intricacies beyond the Heisenberg chain model [11]. Usually, RHCs are obtained as solid solutions of two prototypical spin-chain compounds with different physical properties. A well-known example is $\mathrm{Sr}_{3} \mathrm{CuPt}_{1-x} \mathrm{Ir}_{x} \mathrm{O}_{6}$ [12], whose bulk properties match the real-space renormalization group predictions. To obtain a direct microscopic insight on the problem, we employed NMR experiments to study the local magnetic relaxations in a similar system, namely, $\mathrm{BaCu}_{2}\left(\mathrm{Si}_{0.5} \mathrm{Ge}_{0.5}\right)_{2} \mathrm{O}_{7}$ [13]. In this Letter, we show that a strongly temperature-dependent distribution of magnetically inequivalent local relaxations indeed exists in an RHC material. In the context of the above-mentioned decimation procedure, we regard this phenomenon as an indication of the formation of an RS state.

The material $\mathrm{BaCu}_{2}\left(\mathrm{Si}_{0.5} \mathrm{Ge}_{0.5}\right)_{2} \mathrm{O}_{7}$ considered here is isostructural with its parent $\mathrm{Si}$ and $\mathrm{Ge}$ compounds. The quasi-1D system $\mathrm{BaCu}_{2} \mathrm{Si}_{2} \mathrm{O}_{7}$ (see Fig. 1) has an inchain coupling $J=24.1 \mathrm{meV}$ [14] and orders antiferromagnetically at $T_{N}=9.2 \mathrm{~K}$ [15]. Bond randomness in $\mathrm{BaCu}_{2}\left(\mathrm{Si}_{0.5} \mathrm{Ge}_{0.5}\right)_{2} \mathrm{O}_{7}$ is introduced via the different bond angles of $\mathrm{Cu}-\mathrm{O}$ exchange paths, a direct consequence of the different $\mathrm{Si}$ and Ge covalent radii. A previous study [16] revealed a logarithmic temperature dependence of the spin susceptibility $\chi(T)$ at low temperatures [1], a characteristic

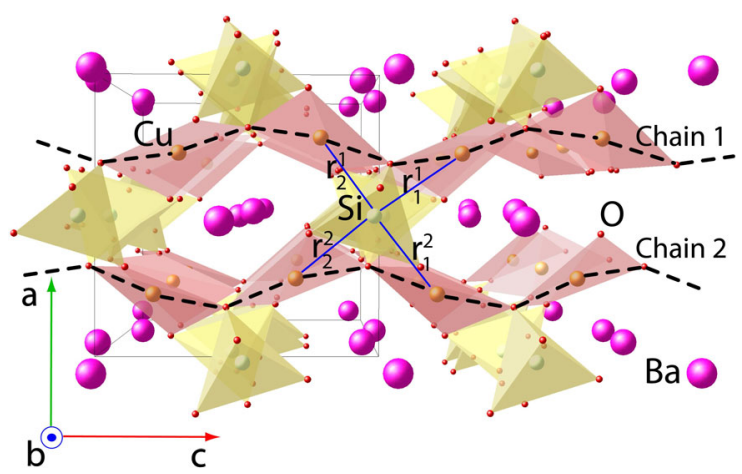

FIG. 1 (color online). Crystal structure of $\mathrm{BaCu}_{2} \mathrm{Si}_{2} \mathrm{O}_{7}$ emphasizing the copper chains (dashed lines) and the nearest neighbor configuration of the silicon atoms. 
RHC feature predicted to be independent of the initial $J$ distribution. Despite that, and following some original misinterpretation, inelastic neutron-scattering experiments were found to be quantitatively consistent with Luttingerliquid behavior expected for a disorder-free spin chain with a single effective coupling $J_{\text {eff }}=\left(J_{\mathrm{Si}}+J_{\mathrm{Ge}}\right) / 2 \approx 37 \mathrm{meV}$ $[16,17]$. It was concluded that the RS-related physics manifests itself at energies much lower than those accessed by neutron studies. Probing these lower-energy or longertime scales with NMR was the main motivation of the present work.

High-quality single crystals of $\mathrm{BaCu}_{2}\left(\mathrm{Si}_{1-x} \mathrm{Ge}_{x}\right)_{2} \mathrm{O}_{7}$ with $x=0$ and $x=0.5$ were grown by the floating zone technique. The magnetic susceptibility was measured via dc SQUID magnetometry. Spin-lattice relaxation times $T_{1}$ were measured by using standard pulse techniques. Because of the rather long relaxation times (tens of seconds at low temperatures), an aperiodic saturation recovery with an echo detection was chosen. With a field $\mu_{0} H=7.024 \mathrm{~T}$ applied along the $a$ axis, the ${ }^{29} \mathrm{Si}$ NMR signal was found at $59.414 \mathrm{MHz}$.

That it is precisely the low-energy dynamics of $\mathrm{BaCu}_{2}\left(\mathrm{Si}_{0.5} \mathrm{Ge}_{0.5}\right)_{2} \mathrm{O}_{7}$ which is most affected by randomness has a clear confirmation in the measured bulk magnetic response (Fig. 2). Unlike for disorder-free spin chains (light dashed lines), the magnetization $M(T)$ of the disordered material (dots) tends to diverge at low temperatures. This divergence is notably different from that observed for $x=1$, where it is related to weak ferromagnetism and thus strongly orientation-dependent [18]. In our case, instead, the $M(T)$ curves only reflect the weak anisotropy of the $g$-factor tensor. We interpret the $x=0.5$, low- $T$ magnetization by the RS theory, which attributes it to the lowenergy states associated with the above-mentioned weakly bound singlets. It predicts $\chi(T) \sim T^{-1} \ln ^{-2}(\Omega / T)$ [2],
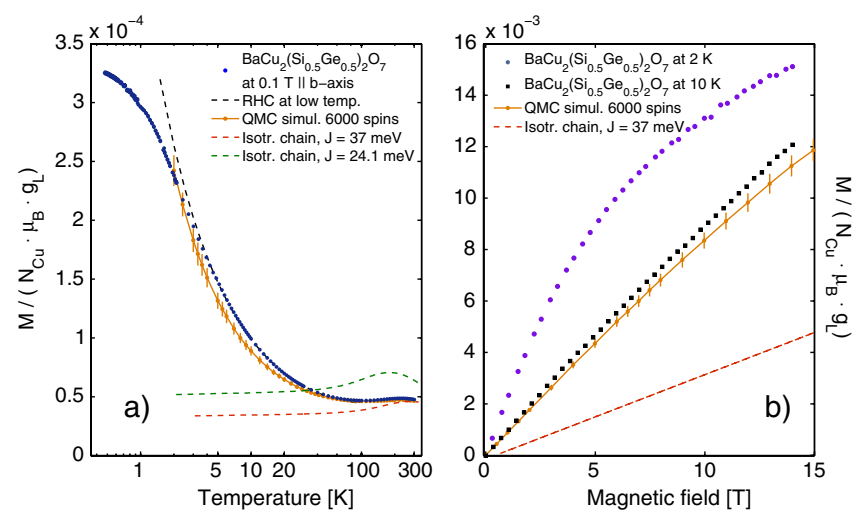

FIG. 2 (color online). Magnetization $M$ per $\mathrm{Cu}$ ion of $\mathrm{BaCu}_{2}\left(\mathrm{Si}_{0.5} \mathrm{Ge}_{0.5}\right)_{2} \mathrm{O}_{7}$ vs temperature (a), and field (b), in normalized units, assuming $g_{L}=2$. The fit of $M(T)$ with an RHC model was adopted from Ref. [2], while the $M(T)$ and $M(H)$ curves for chains with a single isotropic exchange value were calculated as in Ref. [29]. QMC simulations of RHCs are also shown (see text). The symbols are explained in the panels. providing a good fit to the data above $4 \mathrm{~K}$ with a cutoff $\Omega \simeq 66.3 \pm 0.7 \mathrm{meV}$ (dark dashed line). Since the iterative decimation procedure suppresses the strongly bound singlets with an effective coupling larger than $T$, the fraction of unpaired spins is $n_{T} \sim 1 /[\ln (\Omega / T)]^{2}$. The paramagnetism of this fraction implies $\chi(T) \sim n_{T} / T$, with $\Omega$ the temperature above which the entire chain is effectively in a paramagnetic regime. The characteristic energy scale of the disorder-induced excitations is, however, much lower than $\Omega$, because already a $5 \mathrm{~T}$ magnetic field seems to saturate them. This is clearly evidenced by the slope of $M(H, 2 \mathrm{~K})$, which at higher fields tends to that of a disorder-free chain [Fig. 2(b)]. Quantum Monte Carlo (QMC) simulations of Heisenberg spin-1/2 chains, using 6000 spin sites with randomly distributed but equally probable $J_{\mathrm{Si}}$ and $J_{\mathrm{Ge}}$ couplings, confirm these results. $M(T)$ and $M(H)$ averaged over many $(N>40)$ random configurations were obtained via a directed-loop algorithm [19] within the ALPS 2.0 package [20] and are shown in Fig. 2. The $M(H)$ comparison is made at $10 \mathrm{~K}$, where the system can be treated as 1D. Considering the lack of any free parameters, the agreement with the data is remarkably good. Incidentally, a fit of the RS prediction to the low- $T$ QMC results provides $\Omega \approx J_{\mathrm{Ge}}$, thus reinforcing the pertinence of the RS theory.

Similarly to the magnetization data, also ${ }^{29} \mathrm{Si} \mathrm{NMR}$ relaxation rates $T_{1}^{-1}$ for $x=0$ and $x=0.5$ show remarkable differences (see Fig. 3). The local magnetic field at the silicon nuclei originates from the $\mathrm{Cu}$ electronic spins $\mathbf{S}_{j}$ and the relevant hyperfine interactions (see Fig. 1). Provided a matrix description of the latter is adequate and the hyperfine coupling tensor at the $j$ site of the $c$ chain is $A^{j, c}$ (see Fig. 1 for the index notation), the $q$-space quantities $\quad \tilde{A}_{\alpha, \beta}^{c}(\mathbf{q})=\sum_{j=1,2} e^{i \mathbf{q} \cdot \mathbf{r}_{j}^{c}} A_{\alpha, \beta}^{j, c}$, with $\alpha, \beta=\{x, y, z\}$ determine the longitudinal relaxation rate:

$$
\frac{1}{T_{1 z}} \propto \hbar^{2} k_{B} T \gamma_{n}^{2} \sum_{\substack{\alpha=x, y, z \\ q}}\left[\tilde{A}_{x \alpha}^{2}(\boldsymbol{q})+\tilde{A}_{y \alpha}^{2}(\boldsymbol{q})\right] \frac{\chi_{\alpha \alpha}^{\prime \prime c}\left(\boldsymbol{q}, \omega_{n}\right)}{\omega_{n}},
$$

where $\omega_{n}$ is the resonance frequency. Detailed calculations reported elsewhere [21] show that the $\boldsymbol{q}$-dependent prefactor in Eq. (1), also termed the NMR form factor, can be expressed as $f_{\alpha}^{2}(\boldsymbol{q})=M_{\alpha}+P_{\alpha} \cos \left[\boldsymbol{q} \cdot\left(\boldsymbol{r}_{2}-\boldsymbol{r}_{1}\right)\right]$, with $M_{\alpha}$ and $P_{\alpha}$ as constants.

The $\boldsymbol{q}$ sum in (1) was evaluated numerically by using the dynamical susceptibility of an isotropic spin- $1 / 2$ Heisenberg chain given by theory [22]. Because of the large exchange coupling $\left(J=24.1 \mathrm{meV} \gg \mu_{B} B \approx\right.$ $0.4 \mathrm{meV}$ ), spin chains in $\mathrm{BaCu}_{2} \mathrm{Si}_{2} \mathrm{O}_{7}$ were considered as noninteracting above the ordering temperature and effectively in zero field. By employing the simplest linear description of a Luttinger liquid, i.e., without any scattering of quasiparticles and neglecting the influence of the form factor, the dominant contribution in (1) is at $q=\pi$ and $T_{1}$ is temperature-independent. It turns out, however, 
that including $f_{\alpha}^{2}(\boldsymbol{q})$ is essential to obtain a reasonable fit to the $T_{1}^{-1}$ data for $x=0$. Considering $f_{\alpha}^{2}(\boldsymbol{q})$ results in an increase of $T_{1}^{-1}$ with temperature, as observed and shown in Fig. 3. A similar increase, without the influence of $f_{\alpha}^{2}(\boldsymbol{q})$, may also be due to a nonvanishing scattering of quasiparticles, implying that the dominant contribution is at $q=0$, as shown in Ref. [23]. A convincing demonstration of the different implications of $q=0$ and $q=\pi$ excitations on the temperature variations of $T_{1}^{-1}$ was given in Ref. [24] for $\mathrm{Sr}_{2} \mathrm{CuO}_{3}$. Additional data of $T_{1}^{-1}$ with different crystal orientations (not shown here because of space restrictions) confirm that $f_{\alpha}^{2}(\boldsymbol{q})$ cannot be neglected in our case. This fact and the verified absence of significant spin-diffusion effects on the field dependence of $T_{1}^{-1}$ indicate that the dominant component is at $q=\pi$. In Fig. 3, we display the experimental results together with fits considering the two possibilities mentioned above. Including $f_{\alpha}^{2}(q)$ and presuming the zero-field limit, only $J, M$, and $P$ (the latter two independent of $\alpha$ ) are required as free parameters for the fits. The best results are obtained with $J \simeq 55 \mathrm{meV}$, distinctly larger than expected. We recall, however, that the evaluation of $J$ via NMR is not straightforward. It enters via a nonzero form factor, and its uncertainty depends on the choice of the temperature range of the fit. Nevertheless, the results for the $x=0$ case show that ${ }^{29} \mathrm{Si}$ NMR clearly probes the Luttinger-liquid physics, hence providing a convenient technique for studying the low-energy excitations also for $x>0$.

Referring to the latter case, we notice that, due to the loss of translational invariance in disordered systems, Eq. (1) is not valid. The translational inequivalence of sites introduces a multitude of local nuclear relaxation times. For a nuclear spin $I=1 / 2$, the individual relaxations are simply exponential, but collectively they give rise to a systemdependent and generally nonexponential relaxation. If $\tau$ is one of the local relaxation times, a possible interpretation for the observed stretched exponential recovery is provided by $[25,26]$

$$
\int_{0}^{\infty} \rho(\tau, T) e^{-[t / \tau(T)]} d \tau=e^{-\left[t / \tau_{s}(T)\right]^{\beta(T)}} .
$$

This equation implies that if the same type of relaxation occurs with a probability $\rho(\tau, T)$ and with a characteristic temperature-dependent $\tau(T)$ at different nuclear sites, then the global relaxation function is a stretched exponential, characterized by the parameters $\beta(T)$ and $\tau_{s}(T)$. Relaxations described by a stretched exponential are quite common in disordered magnets. The temperature dependence of the exponent $\beta$ has been reported for a number of different systems [27], such as spin glasses, quasicrystals, polymers, etc. In our case, while for $x=0$ a simple exponential is adequate for describing the magnetization recovery, stretched exponentials are needed for $x=0.5$. The corresponding data and fits are shown in Fig. 3(b) and $3(\mathrm{c})$, respectively. From the latter, $\tau_{s}(T) \equiv T_{1}$ and $\beta(T)$ are obtained and shown in Figs. 3(a) and 3(d).

Extracting the weights $\rho(\tau, T)$ via an inversion of Eq. (2) is a mathematically nontrivial task [26]. To account for our case, where $\beta \geq 0.5$, we start from Eq. (2) but now use the variable $s=\tau_{s} / \tau$ [28]:

$$
e^{-\left(t / \tau_{s}\right)^{\beta}}=\int_{0}^{\infty} P(s, \beta) e^{-s t / \tau_{s}} d s,
$$

where $P(s, \beta)$, as $\rho(\tau)$ before, represents a particular probability distribution function (PDF) of relaxation rates for a given value of $\beta$. The inverse of the Laplace transform of (3) [28] provides the required relation between $P$ and $\rho$, the probability distributions of relaxation rates and times, respectively:

$$
\rho\left(\frac{\tau}{\tau_{s}}, \beta\right)=P\left(\frac{\tau_{s}}{\tau}, \beta\right) \frac{\tau_{s}}{\tau^{2}} .
$$

Although $P(s, \beta)$ is obtained only numerically, Eq. (4) allows us to extract the distribution of the local relaxation times in a random Heisenberg chain starting from the experimental NMR data. Results of this procedure for a
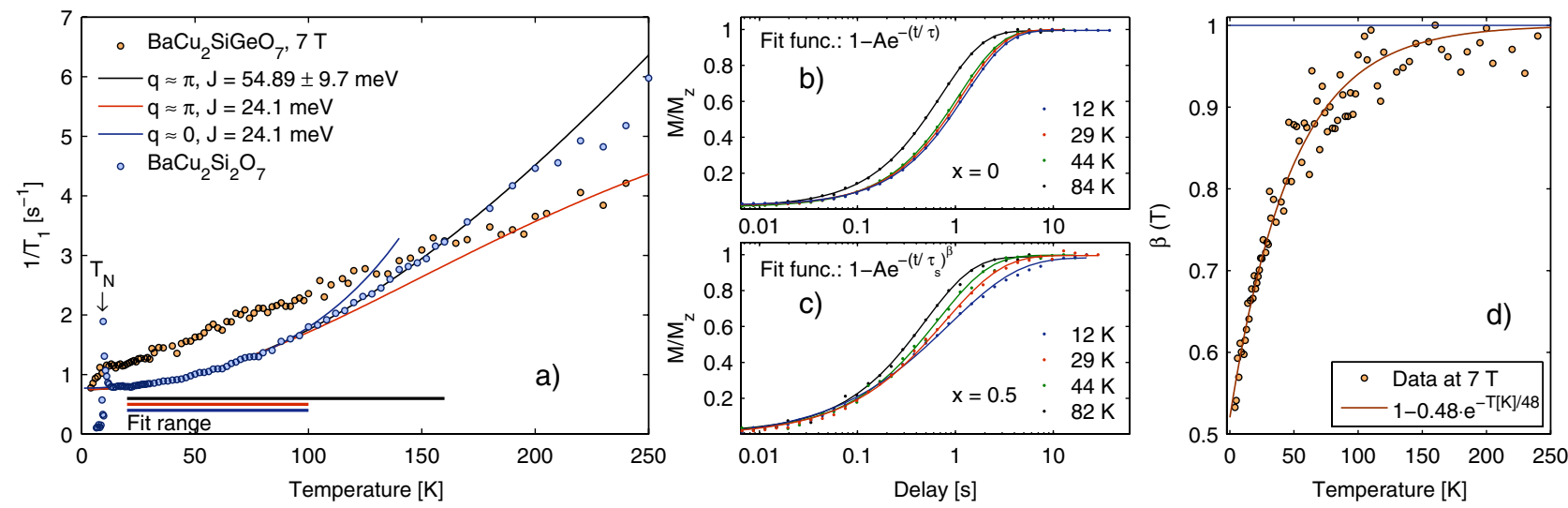

FIG. 3 (color online). ${ }^{29} \mathrm{Si}$ NMR spin-lattice relaxation data of $\mathrm{BaCu}_{2}\left(\mathrm{Si}_{1-x} \mathrm{Ge}_{x}\right)_{2} \mathrm{O}_{7}$ for $x=0$ and $x=0.5$ (no and maximum randomness, respectively). (a) Relaxation rates for both cases, (b),(c) recovery of magnetization, and (d) temperature dependence of $\beta$ for $x=0.5$. The symbols are explained in the panels. 


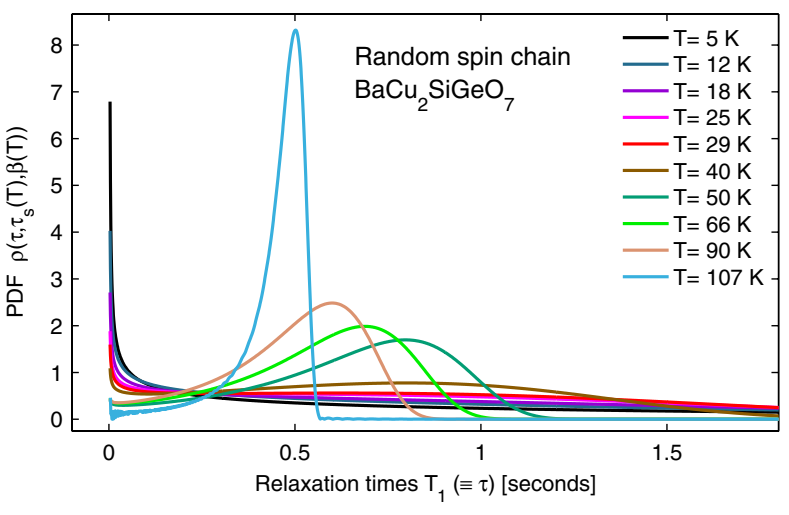

FIG. 4 (color online). Probability distribution of NMR spinlattice relaxation times in $\mathrm{BaCu}_{2}\left(\mathrm{Si}_{0.5} \mathrm{Ge}_{0.5}\right)_{2} \mathrm{O}_{7}$ at different temperatures, as derived from fits to the experimental data partly shown in Fig. 3(c).

series of different temperatures are shown in Fig. 4. At high temperatures, where $\beta \rightarrow 1$, the distribution of relaxation times tends to a delta function peaked at $\tau=\tau_{s}(T)$, while at low temperatures the distribution diverges weakly as $\tau \rightarrow 0$ and hence remains integrable [26]. The integrals of the normalized distributions are temperatureindependent, thus reflecting the conserved total number of magnetic sites. In a disordered system the sites are magnetically inequivalent, giving rise to a broadening of the relaxations' distribution at low temperatures. For $T \rightarrow 0$, the distribution $\rho(\tau, \beta)$ peaks at $\tau \rightarrow 0$, but its mean value $\tau_{s}$ increases due to the longer tail of the distribution.

We argue that the measured NMR parameters can be used to obtain information on the low-energy dynamics of RHC systems, inaccessible by standard neutron-scattering techniques [16]. In the case of randomness, the lack of translational invariance prompts for models based on realinstead of reciprocal-space configurations. In the RS picture, upon lowering the temperature there is an increase in the number of singlets with different couplings being formed, while $n_{T}$ goes to zero. This results in a continuous distribution of magnetically inequivalent sites and in longer $\tau_{s}(T)$ at lower temperatures, as reflected in our data. In this low- $T$ scenario low-energy dynamics is determined by the increasing importance of the frozen singlets, at the expense of the yet uncoupled spins, a point we will address in a forthcoming publication [21].

In conclusion, we have carried out a detailed ${ }^{29} \mathrm{Si} \mathrm{NMR}$ study of the spin-lattice relaxation rates in the $\mathrm{BaCu}_{2}\left(\mathrm{Si}_{1-x} \mathrm{Ge}_{x}\right)_{2} \mathrm{O}_{7}$ system for $x=0$ and $x=0.5$, corresponding to no and maximum disorder, respectively. NMR turns out to be an appropriate technique for the study of low-energy dynamics in gapless antiferromagnets with bond randomness. Guided by the experimental observation that the relaxation of magnetization is characterized by a stretched exponential, we show that it is possible to extract the distribution of relaxation functions in a random
Heisenberg chain from experiment. With the additional evidence from magnetization data and QMC calculations, we interpret this result to reflect the formation of randomsinglet states in a random Heisenberg chain, as predicted by theory.

The $\mathrm{BaCu}_{2} \mathrm{SiGeO}_{7}$ crystals used in this work were prepared by Dr. T. Masuda in the group of Prof. K. Uchinokura at the University of Tokyo. The authors thank T. Giamarchi and P. Bouillot for useful suggestions concerning the NMR form factor. This work was financially supported in part by the Schweizerische Nationalfonds zur Förderung der Wissenschaftlichen Forschung (SNF) and the NCCR research pool MaNEP of SNF.

*fcasola@phys.ethz.ch

[1] C. Dasgupta and S.-K. Ma, Phys. Rev. B 22, 1305 (1980).

[2] D. S. Fisher, Phys. Rev. B 50, 3799 (1994).

[3] E. Westerberg et al., Phys. Rev. B 55, 12578 (1997).

[4] R. A. Hyman and K. Yang, Phys. Rev. Lett. 78, 1783 (1997); C. Monthus, O. Golinelli, and Th. Jolicoeur, Phys. Rev. B 58, 805 (1998).

[5] R. Mélin et al., Phys. Rev. B 65, 104415 (2002).

[6] Y.-C. Lin et al., Phys. Rev. B 68, 024424 (2003).

[7] R. N. Bhatt and P. A. Lee, Phys. Rev. Lett. 48, 344 (1982).

[8] O. Motrunich, K. Damle, and D. A. Huse, Phys. Rev. B 63, 134424 (2001).

[9] E. Yusuf and K. Yang, Phys. Rev. B 72, 020403(R) (2005).

[10] L. C. Tippie and W. G. Clark, Phys. Rev. B 23, 5854 (1981).

[11] A. W. Sandvik, D. J. Scalapino, and P. Henelius, Phys. Rev. B 50, 10474 (1994).

[12] T. N. Nguyen, P. A. Lee, and H.-C. zur Loye, Science 271, 489 (1996).

[13] Y. Yamada, Z. Hiroi, and M. Takano, J. Solid State Chem. 156, 101 (2001).

[14] A. Zheludev et al., Phys. Rev. B 65, 014402 (2001).

[15] I. Tsukada et al., Phys. Rev. B 60, 6601 (1999).

[16] T. Masuda et al., Phys. Rev. Lett. 93, 077206 (2004); 96, 169908(E) (2006).

[17] A. Zheludev et al., Phys. Rev. B 75, 054409 (2007).

[18] I. Tsukada et al., Phys. Rev. B 62, R6061 (2000).

[19] F. Alet, S. Wessel, and M. Troyer, Phys. Rev. E 71, 036706 (2005).

[20] B. Bauer et al. (ALPS Collaboration), arXiv:1101.2646.

[21] F. Casola et al. (to be published).

[22] H. J. Schulz, Phys. Rev. B 34, 6372 (1986).

[23] J. Sirker, R. G. Pereira, and I. Affleck, Phys. Rev. Lett. 103, 216602 (2009).

[24] K. R. Thurber et al., Phys. Rev. Lett. 87, 247202 (2001).

[25] E. W. Montroll and J. T. Bendler, J. Stat. Phys. 34, 129 (1984).

[26] C. P. Lindsey and G. D. Patterson, J. Chem. Phys. 73, 3348 (1980).

[27] J. C. Phillips, Rep. Prog. Phys. 59, 1133 (1996).

[28] D. C. Johnston, Phys. Rev. B 74, 184430 (2006).

[29] D. C. Johnston et al., Phys. Rev. B 61, 9558 (2000). 